\title{
PRIMEROS RESULTADOS DE UN ESTUDIO PSICOPEDAGÓGICO DEL AUTOCONCEPTO EN NIÑOS CON HABILIDADES SOBRESALIENTES
}

Gabriela de la Torre García

\section{RESUMEN}

En la actualidad, una de las principales preocupaciones es la del autoconcepto, tanto es así que han surgido una gran cantidad de herramientas para su evaluación y varios programas de mejora dirigidos a este constructo propiamente humano. La hetereogenidad que caracteriza al grupo de los niños con habilidades sobresalientes, nos presenta este nuevo campo de investigación. El presente escrito busca conocer el autoconcepto de un grupo específico de niños con habilidades sobresalientes, atendidos a través de un programa de enriquecimiento dentro de la línea de investigación de la superdotación de la Universidad Complutense de Madrid ${ }^{1}$.

La Sociedad Española para el Estudios de la Superdotación, dirigido por la Dra. Luz F. Pérez, en conjunto con la Institución Educativa SEK- Santa Isabel, crean un programa de enriquecimiento para niños con altas capacidades. Éste cuenta con un fuerte apoyo científico y de investigación psicopedagógica, avalado por el Departamento de Psicología Evolutiva y de la Educación de la Universidad Complutense de Madrid -con una amplia trayectoria científica y de investigación psicopedagógica en este campo-, y con el apoyo de otras universidades y prestigiosos centros internacionales especializados. El Programa Estrella se concreta, actualmente, en un programa de enriquecimiento psicopedagógico y de apoyo familiar. Se atienden niños desde los 5 hasta los 16 años de edad, en las diferentes áreas de desempeño. Se busca desarrollar integralmente, no sólo al niño con alta capacidad, sino dar atención a la familia en conjunto. 


\begin{abstract}
Today, one of the principal worries is self concept, so is it that they have emerged a high quantity of tools to measure it, and several programs for its improvement of this construct of buman nature. The variety that describe the group of gifted kids, has presented us this new research area. This article pretends to know the self concept of a specific group of gifted and talented children that has been attended by an enrichment program under the research team for giftedness of the Universidad Complutense de Madrid.
\end{abstract}

Palabras clave: Altas capacidades; autoconcepto; autoestima; medición del autoconcepto.

\title{
INTRODUCCIÓN
}

El despliegue de la personalidad es una de las bases para el desarrollo humano integral. El autoconcepto nos permite adquirir una mejor o peor proyección de nosotros mismos, que se refleja no sólo en nuestro bienestar sino el propio desempeño en los distintos ámbitos en los que nos desenvolvemos.

Este escrito pretende realizar un análisis del autoconcepto en los niños con altas capacidades, partiendo de dos tendencias actuales: la que afirma que estos niños poseen un autoconcepto positivo y la segunda que afirma que éste es negativo.

Se estudia la naturaleza de los niños con altas capacidades, desde una definición clara e inclusiva, estableciéndose las diversas áreas de desarrollo del niño con altas capacidades, incluida la referida al conocimiento de uno mismo. Y por último, se revisan las diversas características observadas en 
niños con altas capacidades, teniendo en cuenta que cada uno es diferente y, por tanto, no presentan las mismas particularidades.

El trabajo tiene como objetivo la medición y comparación del autoconcepto en un grupo pequeño de niños con altas capacidades. Se busca comprobar cuál de las dos teorías existentes es cierta en esta población.

Agradecemos la colaboración de la Sociedad Española para el Estudio de la Superdotación.

\section{EL AUTOCONCEPTO}

Sin duda, el autoconcepto es una noción que está en boca de muchas personas desde hace algunas décadas. A partir de los problemas sociales vividos en los últimos años, el desarrollo adecuado del ser humano ha sido el objetivo de muchos programas educativos. No es que en épocas pasadas no se considerara al autoconcepto como contenido del currículo educativo, sino que éste no se hacía de forma expresa, ya que se pensaba que cualquier persona desarrollaba su propio autoconcepto de manera "natural".

Para estudiar el autoconcepto debemos partir de una definición. Siguiendo a un clásico como Raimy (1948), encontramos que el autoconcepto de la persona es: la idea de ella misma desde su punto de vista. No se trata de una mera conglomeración de conceptos aislados sobre la persona, sino de una interrelación diseñada o gestada de todos ellos.

En esta definición no sólo se habla de la visión propia de la persona acerca de ella misma, sino de esa gran cantidad de 
concepciones que la persona tiene de sí misma y que confluyen en una sola imagen.

«El autoconcepto, como otros conceptos, es un conjunto de reglas para procesar información; este conjunto particular tiene la función central de regular y gobernar toda la información procesada y monitorear la entrada sensorial" ${ }^{2}$.

En esta definición encontramos la finalidad del autoconcepto, es decir, aquella que a la propia persona le permite regular, organizar, jerarquizar y utilizar la información que le entra del exterior, de acuerdo a su propio ser y a lo que cree, piensa y proyecta. De no existir un autoconcepto, la persona tendría una gran cantidad de información que simplemente fluiría a través de su ser, sin ser capaz de acomodarla y utilizarla.

La clave del autoconcepto es que no siempre se ha formado en un sentido positivo, debido a que el autoconcepto tiene una importante connotación emocional que marca la formación de nuestra propia idea de nosotros mismos. Por lo que las experiencias que hemos vivido y el ambiente que nos rodea, pueden favorecer un autoconcepto negativo que, entonces, se verá reflejado en nuestra actuación.

Aunque las personas -a excepción de aquéllas con trastornos específicos-, tenemos una identidad única, es cierto que esta identidad está formada por una amplia gama de autoconceptos, que guardan relación con las percepciones y 
los roles específicos que desempeñamos a lo largo de nuestra vida.

Esta cantidad de funciones, roles e ideas que tenemos sobre nosotros, nos llevan a crear una identidad. Por lo que es importante apreciar la relevancia de la visión de los otros en la formación de nuestro propio autoconcepto.

Con la información citada, puede construirse una definición propia del autoconcepto, que integra los conceptos anteriores.

El autoconcepto es un constructo que integra la visión que la persona tiene sobre si misma, dependiendo de su propia noción de sí mismo y de la información que recibe de los demás; y tiene como finalidad regular la información que se adquiere del medio.

Existen dos elementos básicos del autoconcepto. El primero es el conocimiento de uno mismo, que es lo que conocemos como la autoimagen ${ }^{3}$; ésta, se refiere a uno de los elementos a través de los cuales construimos la idea que tenemos de nosotros mismos y de cómo queremos ser: es decir, nuestro ideal o cómo deseamos ser; el segundo es la evaluación que hacemos de nosotros mismos y que es la llamada autoestima.

A pesar de conocerse por este nombre no son equivalentes; la autoimagen es sólo un elemento de la identificación de sí mismo. 
"Los científicos sociales aluden a las percepciones del self distinguiendo dos aspectos:

" - por un lado el autoconcepto o la identificación de sí mismo; " - por otro, la autoestima que se refiere a los sentimientos de estima de sí mismo (Lawson et al., 1979, Wylie, 1961, 1968, 1974, 1979).

"El autoconcepto más general incluye una identificación de las características del individuo, así como una evaluación de las mismas. La autoestima hace más hincapié en el aspecto de las características" ${ }^{4}$.

Es decir, que la autoestima es la valoración -alta o bajaque hacemos de la información procesada por el autoconcepto; por ello, es claro que el autoconcepto comprende un campo de acción más amplio que la autoestima.

En este sentido, podemos decir que la persona tendrá una autoestima más elevada en cuanto más satisfecha esté consigo misma. Lo más relevante es que esto no sólo influye en su propia visión, sino también en la confianza y en la manera en que enfrentará el futuro.

James grafica estas ideas en una pequeña fórmula que aclara y simplifica la noción de autoestima:

$$
\text { Autoestima }=\frac{\text { Éxito }}{\text { Pretensiones... }}
$$

4 Oñate, P., El autoconcepto., p.77. 
En esta pequeña fórmula, podemos medir la manera en que las personas se evalúan. El resultado son esos juicios que la persona hace sobre sí misma y sobre su actuar. Sin embargo, el juicio es diferente en cada una, no sólo porque cada una es única, sino porque las normas y criterios con base en los que se evalúa, son diferentes y están influidos por el medio y por el propio desarrollo.

Para evaluarnos, nos comparamos con la autoimagen que hemos formado, es decir, esa persona que queremos ser, a la que pretendemos llegar, día a día, con nuestras acciones e ideas. Después nos comparamos con los juicios que los otros hacen sobre nosotros; nos damos cuenta de cómo nos perciben y lo integramos en nuestra evaluación. Por último, realizamos una comparación entre lo que hemos hecho y el grado en que hemos alcanzado nuestro ideal.

El autoconcepto no depende sólo de nosotros, sino de la información que nos es dada sobre nosotros mismos, el medio que nos rodea. Por lo que: «El autoconcepto no es innato, sino que se desarrolla a partir de una mira de experiencias diferentes y acumulables que entrañan al "yo", al "mi" y a lo "mío" 5.

Es decir que no nacemos con una idea de nosotros mismos, sino que la vamos desarrollando con la información que iremos acumulando a lo largo de la vida. El conocer nuestra propia persona nos da la potencialidad de planear el futuro y, así, ir viviendo nuevas experiencias que nos continúan 
formando día a día. También nos planteamos distintas expectativas de vida, de acuerdo a nuestra propia noción.

Resaltemos estas características en la infancia, que es la etapa evolutiva que nos ocupa:

"El sentido de sí mismo crece en forma lenta. Comienza en la infancia con la autoconciencia (...). Alrededor de los 18 meses, tiene su primer momento de autorreconocimiento, cuando se reconoce a sí mismo en el espejo. El siguiente paso es la autodefinición. Esto sucede cuando identifica las características que considera importantes para describirse él mismo. A los tres años de edad, se juzga principalmente en término externos (...). Sólo a los seis o siete años de edad (...) empieza a definirse en términos psicológicos. Entonces es cuando desarrolla el concepto de quién es (el yo verdadero) y también de quién le gustaría ser (el yo ideal)" ${ }^{6}$.

Burns distingue cinco fuentes del autoconcepto; cada una de éstas se desarrolla en distintas etapas evolutivas, pero todas inician en la infancia:

1. Imagen corporal.

2. Lenguaje.

3. Interpretación de la formación procedente del medio ambiente.

4. Identificación del rol sexual.

5. Prácticas educativas con los niños.

La primera, imagen corporal, se desarrolla desde el momento en el que nacemos y está ligada con la apariencia

Papalia, D., Psicología del desarrollo de la infancia a la adolescencia., p. 459 
física. Primero distinguimos nuestro cuerpo y después lo vamos adjetivando. Esta noción de nuestro físico va cambiando a lo largo de la vida, pero tenemos una idea básica de cómo somos físicamente ${ }^{7}$.

La segunda es el lenguaje, en donde se produce un cambio vital en los primeros años de vida, es decir, nos reconocemos como un "yo", y podemos diferenciarnos de un "tú". Aunque no en todos los niños surge este conocimiento en el mismo momento, es notorio cuando son capaces de diferenciarse de los otros.

La interpretación de lo exterior, que es la tercera fuente, es quizás la más compleja, pero la más cotidiana. Continuamente valoramos lo que los demás opinan de nosotros y aunque se origina en diversas medidas en cada persona es fundamental, para nuestra propia concepción, conocer e integrar lo que los otros piensan y, en especial, quienes me son significativos.

El cuarto supone un período más avanzado de la vida, aunque comienza en la niñez; la identificación con un rol sexual, no sólo depende de nosotros, sino de los modelos que hemos tenido y de la formación recibida.

El último, pero no menos importante, es la educación que han recibido los niños, no sólo en la familia, que es el agente educador por excelencia, sino en la escuela. Los estilos de enseñanza utilizados tanto en casa como en la escuela, pueden marcar el desarrollo del propio concepto de un niño.

\footnotetext{
Cfr. Burns., El autoconcepto., p.97.
} 
Para que este autoconcepto se desarrolle de manera positiva sobre la base que hemos establecido, se necesitan dos juicios de la propia persona: el primero es relativo a las cualidades esenciales que se requieren para tener éxito en un área en la que queremos involucrarnos; y el segundo, es el grado en que cada uno siente que posee esas cualidades ${ }^{8}$. Dependiendo de estas dos ideas sobre nosotros mismos, podremos enfrentar una nueva tarea con perspectiva de éxito o de fracaso.

La formación y la dinámica del autoconcepto son complejos; las situaciones que vivimos nos van marcando de una u otra manera y cambian la noción de nosotros mismos en diversas formas; algunas de las ideas que pueden modificar esta noción son: el físico, la moralidad, la ética, nuestras relaciones familiares y sociales, y nuestra personalidad.

En la infancia, todas estas concepciones se van formando y van moldeando nuestro autoconcepto, y en consecuencia la forma en que actuamos y las metas que nos planteamos y que perseguimos.

"Que la conducta humana depende del autoconcepto de los individuos ha sido considerado de forma amplia tanto en la psicología como en la sociología. La investigación ha demostrado que la decisión de una persona de ejecutar ciertas conductas, depende del grado en que el autoconcepto es potenciado o amenazado" ?. 
Esto es verdadero desde la infancia, en donde se puede potenciar o minimizar el autoconcepto de cada niño. La trascendencia de esta formación se traduce en el propio actuar y el propio destino de esa persona.

En la infancia, el autoconcepto positivo no sólo se refleja en el área social del niño, en donde es capaz de establecer mejores relaciones con los demás; un niño con un buen autoconcepto, sabe que es capaz de aprender y reconoce sus limitaciones. Tiene mejores resultados, entre otras razones porque sabe cuándo ha de pedir ayuda para superar sus fallos y potenciar lo que se le facilita de manera natural.

"Los alumnos con un alto grado de autoestima se mostraron más activos y con más éxito, desde el punto de vista social y académico. Expresaban con facilidad sus opiniones en las discusiones y no se contentaban únicamente con escuchar. No eran particularmente susceptibles a las críticas ni les perturbaba demasiado la ansiedad o los problemas psicosomáticos" ${ }^{10}$.

A través de estas aseveraciones, podemos constatar que la autoestima positiva beneficia en varios campos del actuar humano a los niños. Y debemos recordar que mientras se acrecienta la autoestima, se va fortaleciendo en conjunto el autoconcepto, con lo que se esperan decisiones más congruentes con un ideal positivo.

Es importante reconocer que si el autoconcepto no se ve favorecido, pueden empezarse a generar graves conflictos en el niño; conflictos que se reflejarán en su actuar académico, 
social y familiar. Por esta razón, podemos encontrar niños agresivos o con bajo rendimiento escolar.

Para formar un autoconcepto favorable es necesario poseer un equilibrio claro y real de los aspectos que conforman nuestro ideal. Y en la infancia, es vital que tanto padres como maestros colaboren en la tarea de lograr este objetivo.

Las bases que se asienten en la infancia, marcarán el desarrollo del autoconcepto a lo largo de la vida. Por esta razón un autoconcepto sano, equilibrado y favorable nos ayudará en el futuro desarrollo de esa persona.

La razón última por la que es importante que cada niño se desarrolle de manera positiva -en lo referente a su autoconcepto y su persona en general- es el logro de su propia felicidad. Una persona que tiene un autoconcepto positivo y que va acercándose a su yo ideal, alcanzará más fácilmente su propia felicidad. Y si los objetivos que esta persona se plantea son armónicos con los de la propia sociedad en la que se desarrolla, entonces los beneficios no sólo serán para el propio desempeño de la persona, sino para el de la sociedad en general.

\section{MEDICIÓN}

Debido a que el autoconcepto, como otros conceptos humanos, no es tangible, su medición es complicada. Sin embargo, para poder intervenir y mejorar este autoconcepto, o para conocerlo y poder comprender su influencia en otros ámbitos del actuar humano, es necesario medirlo.

Para Burns (1990), existen dos formas de medir el autoconcepto: la primera es a través del método del autoinforme 
y la segunda mediante la observación. Los autoinformes básicamente consisten en que la propia persona dé a conocer, a través de diversas técnicas, lo que piensa sobre sí misma.

Existen varios tipos, dentro de los cuales se hallan:

- Escalas de calificación.

- Listas de calificación.

- Cuestionarios de clasificación.

- Métodos de respuesta no estructurada y libre.

- Técnicas proyectivas.

- Entrevistas ${ }^{11}$.

Estos métodos de autoinforme pueden ser muy válidos, ya que si consiguen aplicarse a grandes segmentos de la población, los resultados pueden compararse para conocer cómo está el autoconcepto de una persona en relación con los demás, o con determinadas variables que queremos medir.

Los métodos de medición a través de la observación son útiles en cuanto a que la calidad de la información puede ser mejor y además observarse una gran cantidad de variables a mayor profundidad. Sin embargo, dependen mucho de la capacidad del evaluador, porque es él quien es responsable de conocer a la persona con la profundad necesaria para poder emitir juicios, y en este proceso interviene una gran cantidad de factores.

La elección de uno u otro método depende de los propios objetivos del evaluador y de las características de la población a evaluar. Existen algunos métodos conocidos y populares 
entre los investigadores, pero no siempre son los más adecuados para el estudio específico que una persona pretende llevar a la práctica.

A pesar de las dificultades que implica la medición del autoconcepto, es verdad que hoy existe una mayor cantidad de métodos y técnicas para evaluar aspectos específicos del autoconcepto, y sería un error no tomarlos en cuenta al momento de realizar una medición.

Cuando medimos el autoconcepto debemos ser conscientes de que es un tema que puede prestarse a manipulación por quien es evaluado. Es importante contar con un mínimo conocimiento de quién está respondiendo a nuestro método de evaluación, si queremos que éste tenga mayor validez.

Conocer el autoconcepto de una persona no sólo nos deja ver las razones por las que actúa como lo hace, sino las posibles conductas que pueda presentar en un futuro.

Dependiendo del camino que la persona siga, será necesario intervenir o no, siempre que sea en beneficio de ésta.

\section{EL NIÑO CON ALTAS CAPACIDADES}

A lo largo de la Historia se ha tratado de definir a quienes nacen con capacidades superiores en cualquiera área de la actividad humana. Aunque siempre ha existido la curiosidad de entender a aquellos que realizar cosas que la mayoría no puede, el estudio sistemático de estas personas es reciente. Sin duda, la definición que muchos países han adoptado, y que es popular, es la de Renzulli, uno de los más grandes teóricos en esta área de la educación especial. Esta definición asegura que: 
"Ser persona con altas capacidades se refiere a la interacción entre tres grupos básicos de rasgos humanos; estos grupos consisten en capacidades generales por encima de la media, altos niveles de compromiso con la tarea y altos niveles de creatividad" ${ }^{12}$.

\section{Definición de altas capacidades de Renzulli}

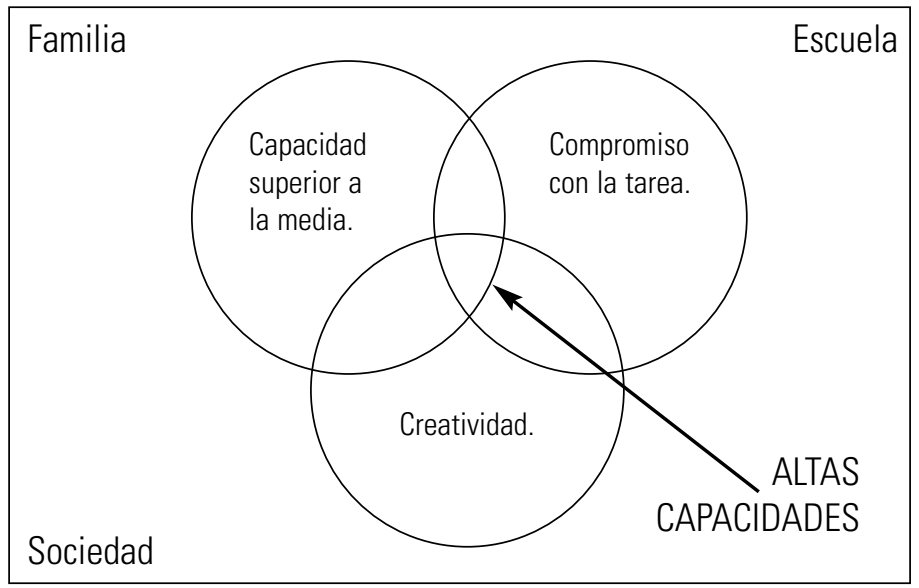

RENZULLI, J.S., What Makes Giftedness?

En Estados Unidos, en 1921, Lewis Terman empezó el primer estudio longitudinal de las personas con altas capacidades. Se incluyeron a 1500 estudiantes que tuvieran un coeficiente intelectual (CI) mayor a 140, a quienes se estudió durante treinta años. Sin embargo, no fue sino hasta 1972 que

12 Renzulli, J., What makes giftdness?, p.34.

* WEBB, J., et al., Guiding the gifted child., p. 49. 
se pidió a Sidney Marland, Secretario de Educación, que elaborara un reporte; a partir de este informe y de los resultados de las investigaciones de Terman, se generaría la definición oficial de la legislación de Estados Unidos, la cual señala que las personas con altas capacidades son:

"Niños, y en su caso jóvenes, identificados en los niveles preescolar, elemental o medio, quienes por tener capacidades, demostradas o en potencia, que evidencian un elevado rendimiento en áreas tales como la intelectual, creativa, académica específica, o liderazgo, o en las artes visuales o escénicas; razón por la cual requieren servicios o actividades que la escuela no proporciona de forma cotidiana" ${ }^{13}$.

Las dos definiciones citadas anteriormente son las más conocidas mundialmente, pero no son las únicas utilizadas para definir al niño con altas capacidades. Sin embargo, las definiciones oficiales son importantes porque de ellas dependen las acciones tomadas en los países respecto a las necesidades de estas personas. Ambas manejan el concepto de capacidad superior, factor común en todas las definiciones existentes de persona con altas capacidades. Pero en la definición de Renzulli, no se establecen las áreas en las que se puede tener un alto desempeño, mientras que en la de Marland hay una división clara de estas áreas.

Existen tantas definiciones de persona con altas capacidades como investigaciones acerca de ellas. Cabe mencionar que las dos definiciones anteriormente mencionadas, engloban a la mayoría de las existentes. Para la autora del presente trabajo:

${ }_{13}$ Leyes públicas 94-142, 99-457 y 101-476, Legislación de Estados Unidos de América. 
La persona con altas capacidades es aquella que muestra una capacidad intelectual superior a la media, se compromete con la tarea y es creativa. Se desarrolla en una o varias áreas del actuar humano; como consecuencia de esto, tiene necesidades educativas especiales que deben ser atendidas para que pueda desarrollarse plenamente.

Una de las dificultades al estudiar niños con altas capacidades es que éste es un término que ha sido definido, en los últimos años, como un término "paraguas", porque caben muchos tipos de niños con altas capacidades dentro de un solo nombre. Es decir, que dentro de este conglomerado, podemos encontrar niños realmente diferentes que expresan su alta capacidad de diversas formas.

Esta realidad se evidenció desde que Whiple, en 1925, definió por primera vez a las personas con altas capacidades, ya que se constató la amplia variabilidad de los modos en que las personas adquieren, organizan, retienen y generan conocimiento, es decir, las diferentes formas en las que se podía sobresalir ${ }^{14}$.

Cada persona con altas capacidades es única; las manifestaciones de sus altas capacidades son diversas. Sin embargo, existen algunas características generales que presentan la mayoría de ellos.

Una de las principales particularidades que definen a la persona con altas capacidades, es la intensidad. Para el doctor Webb ésta es, sin duda, la esencia de estas personas, 
porque todo lo que realizan es "intenso". Así, podemos observar que cuando un niño decide investigar un tema porque le parece interesante y lleva a cabo una investigación tan profunda, puede llegar a parecer una obsesión ${ }^{15}$.

Derivado de esta intensidad, nace el tan famoso "perfeccionismo" que es uno de los atributos que comúnmente se les conoce. Algunas de las bases sobre las cuales descansa este perfeccionismo son los distintos sentimientos generados en torno al "fracaso", es decir, a una falta de perfección; lo que les crea una autocrítica constante.

Una característica más que observamos en la mayoría de las personas con altas capacidades, es la disincronía.

"La disincronía es un término acuñado por J. CH. Terrasier referido a una carencia de sincronización en los ritmos del desarrollo intelectual, afectivo y motor, que suele darse en los niños superdotados. Este desequilibrio afecta a varios aspectos de la vida de estos niños y sus resultados pueden producir problemas de rendimiento, personalidad y sociales ${ }^{16}$.

Estas disincronías dependen de atributos que la persona con altas capacidades no sólo ha heredado, sino de las oportunidades que el ambiente le proporciona para desarrollar aquello que le es dado. Usualmente pensamos que las características que se perciben en varios miembros de una familia son adquiridas genéticamente, pero nos olvidamos que una familia no sólo comparte los genes, sino también el medio

${ }^{15}$ Cfr. Webb. J., et al., Guiding the gifted child., p.47.

${ }^{16}$ Prieto, M., Los superdotados: esos alumnos excepcionales., p.165. 
ambiente. Muchas características de la persona con altas capacidades, son moldeadas en la familia.

Para conocer a la persona con altas capacidades y comprender las características que pueden incluir en su autoconcepto, se han dividido cuatro grandes áreas de estudio del desarrollo humano. Es importante considerar que estas características son específicas, por lo que no se presentan en la mayoría de los niños con altas capacidades.

a) Físicas. Una de las características más importantes que pueden surgir en algunos niños con altas capacidades, es la discrepancia entre el desarrollo físico y el intelectual, mencionado anteriormente. El intelecto del niño quisiera hacer cosas que su cuerpo no le permite. De aquí se deriva una baja tolerancia hacia el retraso en los estándares de las habilidades atléticas.

b) Cognitivas. En general, todas las habilidades cognitivas de estos niños son altas, pero algunas resaltan más que otras. El niño con altas capacidades puede retener y procesar una gran cantidad de información. Esta característica, junto a una comprensión avanzada, hace que el niño con altas capacidades progrese rápidamente en los contenidos escolares. No todos ellos avanzan con la misma rapidez en las distintas áreas; hay algunos que no poseerán la misma capacidad lingüística que matemática, pero en general, pueden manejar todo tipo de información.

Los niños con altas capacidades tienen interés y curiosidad por temas variados e inusuales. No sólo esto, sino que es común que la manera de aproximarse a temas cotidianos sea 
inusual. Como se mencionó anteriormente, los intereses que genera son profundos, esto desemboca en un alto grado de concentración en estos puntos de atracción.

c) Afectivas. Esta área se caracteriza por ser una de las más conflictivas: la famosa característica de la intensidad también se refleja en su afectividad. Generalmente, el niño con altas capacidades posee una gran acumulación de información sobre emociones que no ha sido traída a la conciencia. Esto lo hace contar con una perceptibilidad inusual hacia las expectativas y sentimientos que otros tengan acerca de él.

La mayoría de las personas con altas capacidades muestran una gran preocupación hacia los problemas mundiales. Su sentido de justicia e idealismo aparece en edades muy tempranas y son los iniciadores de esta preocupación. Generalmente se involucran en alguna causa que les lleve a satisfacer esta inquietud.

El niño con altas capacidades tiende a ser una persona que goza de un alto sentido del humor. Muchas de sus tensiones las canaliza a través de esta cualidad. Generan una jerarquía de valores desde una edad temprana y viven de acuerdo a ella, tienen una gran necesidad de una coherencia de vida; se frustran y sufren cuando las personas que los rodean no poseen esta coherencia.

d) Sociales. Por lo general, estos niños saben que son diferentes a los demás, por lo que les es difícil relacionarse. Algunos niños optan por esconder sus habilidades para estar igual que sus compañeros. Necesitan tener aceptación social y pertenecer al grupo. Este problema es aún más agudo con las niñas con altas capacidades. 
En algunos niños, el interés extremo por una tarea hace que pierdan la oportunidad de socializar con otros niños. Prefieren dedicar más tiempo a sus actividades que compartir este espacio con los demás niños.

\section{EL AUTOCONCEPTO EN EL NIÑO CON ALTAS CAPACIDADES}

Los niños con altas capacidades son muy diferentes entre sí, y no podemos afirmar que la relación con los demás siga determinado patrón. Sin embargo, es cierto que como todos los niños, los vínculos que establece con los otros son muy importantes en su desarrollo integral.

En cuanto al desarrollo social, también hay una fuerte influencia tanto del contexto como de la personalidad del niño. En este terreno como ya se mencionó anteriormente, existe diversidad entre los niños con altas capacidades. Una gran cantidad de autores ha encontrado que el desarrollo social de estos niños también es avanzado y que no se generan problemas en esta área, pero esto no es totalmente cierto para todos los niños con altas capacidades.

"(...) los superdotados no constituyen un grupo homogéneo en lo que a socialización se refiere y que, por consiguiente, muestran entre ellos tantas o parecidas diferencias a las observadas en la población normal. Así, por ejemplo, frente al comportamiento socialmente desadaptado de algunos sujetos superdotados, se explicaría la existencia de otros, cuyos elevados índices de popularidad entre sus compañeros dan buena muestra de su capacidad de adaptación al grupo" ${ }^{17}$.

${ }^{17}$ Pérez, L., et al., 10 palabras clave en superdotados., p.67. 
Aunque socialmente estos niños pueden tener un desempeño diverso; existen características que los distinguen: por lo general tienen un sentido del bien y del mal muy claramente delimitado, por lo que tienden a preocuparse por el otro. Esto hace que generen una jerarquía de valores desde muy pequeños e identifica la manera en que establecen sus relaciones; en algunos niños puede observarse una notable madurez al momento de establecer relaciones con los demás.

El autoconcepto es una de las características analizadas con mayor interés en el tema de las altas capacidades, aunque quizás no sea la más estudiada. Sin embargo, algunos especialistas le han dedicado una buena parte de su tiempo. Dentro de los más notables, Renzulli enfatiza que, dentro de las particularidades que definen al niño con altas capacidades, está el autoconcepto.

El autoconcepto en el niño con altas capacidades ha sido un tema muy discutido, debido a que existen dos posturas contrarias que han prevalecido a lo largo de mucho tiempo. Ambas tienen diversas investigaciones que fortalecen su postura.

"Ha habido una discusión de dos categorías de autoconcepto: un autoconcepto académico en el que los estudiantes con altas capacidades por lo general, puntúan muy alto, y el autoconcepto social, un área que es generalmente baja entre los estudiantes con altas capacidades" ${ }^{18}$.

${ }^{18}$ Clark, B., Growing up gifted., p. 239. 
Ésta fue la tendencia que se siguió por algunos años, pero nuevas investigaciones han probado que los niños con altas capacidades poseen una puntuación similar a los niños que se encuentran dentro de la media.

Un ejemplo de estas investigaciones es el de la Universidad de Murcia sobre el autoconcepto y su incidencia en las altas capacidades:

“(...) no se producen diferencias significativas en los factores relativos al autoconcepto personal, referido a la relación e interacción que tiene el superdotado con sus compañeros, padres y profesores. (...) los superdotados, se caracterizan por mostrar un mayor autoconcepto en el campo académico, que sin embargo no se extiende a los ámbitos personal y social, ${ }^{19}$.

También es importante resaltar que existen otros estudios que nos dejan saber más sobre el autoconcepto entre los niños con altas capacidades. Uno de los más relevantes es de Kelly y Colangelo, debido a que encontraron que no hay una variación significativa entre el autoconcepto entre niños y niñas con altas capacidades. Aunque, como ha sido muy estudiado, muchas niñas prefieren esconder sus altas capacidades en determinadas edades, como la adolescencia, cuando la apariencia física parece ser mucho más importante ${ }^{20}$.

Otra variable importante en el desarrollo del autoconcepto de lo niños con altas capacidades se refiere al niño que ha sido etiquetado como tal. Para los niños que han sidos

19 Prieto, M., Identificación, evaluación y atención a la diversidad del superdotado., p.134.

${ }^{20}$ Cfr. Prieto, M., op. cit., p.125. 
etiquetados, la presión parece ser más fuerte, y las expectativas propias y de los demás más altas, lo que afecta su autoconcepto, sobre todo si no llegan a cumplir las expectativas.

Los padres de los niños con altas capacidades influyen en el autoconcepto de su hijo desde el momento en que deciden $-\mathrm{O}$ no- etiquetarlo. Se ha mostrado que los niños no necesitan saber su coeficiente intelectual, pero que deben entender las conductas asociadas con las altas capacidades. Estar consciente de las necesidades y responsabilidades de las altas capacidades, permite al niño examinar sus propias acciones y expectativas y las que tienen de los demás ${ }^{21}$.

Sin embargo, es necesario que el niño comprenda que se le quiere por ser quien es y no por sus altas capacidades. El problema puede iniciarse si se hace demasiado énfasis en sus altas capacidades, no sólo al conversar con él, sino en los comentarios realizados con los demás.

El autoconcepto del niño con altas capacidades será beneficiado o dañado por la relación que establezca con su familia, como primer agente educador, ya que crea en él una imagen de sí mismo que será difícil de modificar. El impacto de los padres es tan fuerte en la vida de los niños que los hace vivir en una constante preocupación al no poder complacerlos; cuando los padres confían en su hijo, y le demuestran que lo quieren por lo que es -y no sólo por su inteligencia-, el niño con altas capacidades genera confianza en sí mismo. 
Además de sus hermanos, el siguiente grupo de personas significativas en la vida del niño con altas capacidades son los amigos; para él, son personas tan importantes como su familia. La mayoría de los niños con altas capacidades tienen amistades largas y duraderas, porque conocen el sentido de la amistad desde pequeños.

No es extraño que el niño con altas capacidades encuentre en la escuela o en otros lugares en los que interactúe con niños, amigos con altas capacidades. Para ellos, estas amistades tendrán especial importancia, ya que les permitirán desarrollar muchos de sus intereses y resolver inquietudes.

La forma en que sus amigos los tratan y los perciben, marca el autoconcepto del niño con altas capacidades. Si estos niños no tienen amigos que los puedan comprender, como lo hace otra persona con altas capacidades, no será extraño que esto afecte a su autoconcepto, haciéndolo sentir alienado y diferente ${ }^{22}$.

Las amistades para el niño con altas capacidades son vitales, por lo que éstas deben procurarse. Para ellos, estos amigos forman parte esencial de su vida y de su desarrollo. Debido a esto, el tener estas amistades fortalecerá el autoconcepto del niño con altas capacidades.

Éstos son los grupos significativos que influyen en el desarrollo del autoconcepto del niño con altas capacidades. En la investigación presente se pretende conocer este desarrollo.

22 Cfr. Clark, B., op. cit., p.245. 


\section{INVESTIGACIÓN DESARROLLADA}

\section{Objetivos}

- Medir el autoconcepto a través de un instrumento válido y confiable.

- Verificar una de las dos posturas concernientes al autoconcepto en los niños con altas capacidades.

- Comparar cinco factores del autoconcepto, enfatizando el factor intelectual.

- Comprobar si el autoconcepto varía entre niños y niñas.

\section{Hipótesis}

El autoconcepto de los niños con altas capacidades, que reciben ampliación curricular, es superior al de la media, sin importar el sexo; y tiende a ser más positivo mientras que el niño va creciendo.

\section{Metodología}

Para llevar a la práctica esta investigación, la autora del presente trabajo aplicó un instrumento de medición del autoconcepto; se eligió la Escala de Autoconcepto de Piers Harris para niños, con un grupo de 11 niños de edades entre los 10 y los 13 años.

\section{Población}

Se eligieron 11 niños del programa de enriquecimiento para niños con altas capacidades, pertenecientes a dos grupos del programa, por lo que su elección es azarosa.

El programa, se desarrolla fuera del horario escolar, fundamentalmente los sábados por la mañana, y está constituido por un conjunto de actividades que realizan los alumnos 
según su edad y necesidades, así como una atención individualizada a las familias que lo solicitan [www.ucm.es/info/sees].

Dentro del programa, los niños se encuentran divididos en siete grupos, dependiendo de sus capacidades y edades, como se mencionó anteriormente. Se eligieron tres grupos que pertenecen a edades que fluctúan de los 10 a los 13 años de edad.

El grupo de niños a los que se examinó, consta de siete hombres y cuatro mujeres. Los niños provienen de diversas escuelas, todos son españoles y, en general, tienen una posición socioeconómica acomodada. Reciben atención a través de este programa de enriquecimiento, y además, cuentan con atención personalizada.

\section{Instrumento de medición}

Para evaluar el autoconcepto de la población se eligió la Escala de Autoconcepto de Piers Harris (1969). La escala consta de 80 frases sencillas con respuesta dicotómica (SÍ-NO); en las que se pide al alumno que decida sí coincide o no, con lo que él piensa. Ello permite obtener una puntuación global y seis dimensiones específicas:

1. Conductual: es la percepción de portarse bien en distinto tipo de situaciones, que el niño parece asociar con la idea general de ser bueno.

2. Intelectual: percepción de ser importante y competente en la situación del aula. Podría identificarse con la percepción del estado socio-académico.

3. Físico: depende de la apariencia y de la percepción de competencia física. 
4. Falta de ansiedad: percepción de ausencia de problemas de naturaleza emocional.

5. Autoconcepto social o popularidad: evalúa básicamente, la percepción que el alumno posee de sus relaciones con los otros niños.

6. Felicidad: satisfacción o autoestima.

La aplicación de esta escala puede hacerse, de forma colectiva, desde el segundo curso de enseñanza primaria.

Se eligió esta escala por diversas razones; dentro de las más importantes:

- Es una escala con un amplio reconocimiento científico.

- Es válida y confiable.

- Es sencilla de usar y fácil de comprender para los niños.

- Los resultados se pueden dividir en seis áreas y no en un solo resultado global.

\section{Resultados Preliminares}

Los resultados globales con las medias correspondientes se muestran en la siguiente tabla:

\begin{tabular}{|l|r|r|r|r|r|r|r|r|r|r|r|l|}
\hline Factores & 1 & 2 & 3 & 4 & 5 & 6 & 7 & 8 & 9 & 10 & 11 & Media \\
\hline Autoconcepto general & 6 & 30 & 67 & 51 & 61 & 57 & 58 & 68 & 76 & 78 & 27 & 52.7 \\
\hline Conductual & 17 & 7 & 18 & 15 & 12 & 15 & 15 & 15 & 18 & 18 & 12 & 13.3 \\
\hline Intelectual & 17 & 9 & 14 & 15 & 13 & 14 & 14 & 13 & 16 & 18 & 6 & 14.8 \\
\hline Físico & 12 & 1 & 6 & 6 & 9 & 5 & 5 & 10 & 11 & 12 & 1 & 7.09 \\
\hline Ansiedad & 8 & 5 & 7 & 7 & 9 & 6 & 12 & 9 & 9 & 11 & 3 & 8 \\
\hline Popularidad & 12 & 3 & 11 & 5 & 10 & 12 & 10 & 11 & 12 & 11 & 2 & 9 \\
\hline Felicidad & 9 & 3 & 9 & 6 & 8 & 6 & 9 & 9 & 9 & 9 & 1 & 6.36 \\
\hline
\end{tabular}


El autoconcepto general de los niños estudiados se encuentra dentro de la media (52.7), siendo ésta de 63.3 con una desviación típica de 10.24. Esto implica que el autoconcepto general de estos niños está de acuerdo con los demás niños de su edad.

En cuanto al análisis de los seis factores que componen esta prueba, podemos distinguir:

- El autoconcepto conductual; que incluye items como: 1. "Se puede confiar en mí"; 2. "Me porto bien en el colegio"; 3. "Me llevo bien con la gente"; 4. "Soy obediente"; 5. "Soy una buena persona", que es de 13.3, está dentro de la media y se sitúa en el percentil 25.

- El autoconcepto intelectual; que incluye items como: 1. "Soy listo"; 2. "Cuando sea mayor voy a ser una persona importante"; 3. "Hago bien el trabajo del colegio"; 4. "Soy un miembro importante de mi clase", que es de 14.8, está dentro de la media y se sitúa en el percentil 50.

- El autoconcepto físico, que incluye ítems como: 1. "Soy guapo"; 2. "Tengo una cara agradable"; 3. "Soy uno de los mejores en juegos y en deporte»; que es de 7.09, está ligeramente por debajo de la media y se sitúa en un percentil 20.

- El autoconcepto de ansiedad, que incluye ítems como: 1. "Me preocupo mucho cuando tenemos un examen en el colegio"; 2. "Duermo bien por la noche"; 3. "Cuando las cosas son difíciles, las dejo sin hacer"; que es de 8, está dentro de la media y se sitúa en el percentil 50. 
- El autoconcepto social o popularidad, que incluye ítems como: 1. "Me resulta difícil encontrar amigos"; 2. "Mis compañeros de clase se burlan de mí"; 3. "Caigo bien en la clase", que es de 9, está dentro de la media y se sitúa en el percentil 30 .

- La felicidad o autoestima; que incluye items como: 1. "Soy una persona feliz"; 2. "Me gusta ser como soy"; 3. "Mi familia está desilusionada conmigo", que es de 6.36, está ligeramente por debajo de la media y se sitúa en el percentil 10.

En una valoración cualitativa podemos argumentar que:

a. De los cuestionarios, hay dos con puntuaciones extremadamente altas y dos con puntuaciones extremadamente bajas. Las dos personas con puntuaciones extremadamente altas son mujeres, mientras que los que tienen puntuaciones muy bajas, uno es hombre y la otra es mujer, además ambas personas se caracterizan por tener capacidades muy altas.

b. El autoconcepto de las mujeres (62.5) de este grupo es más alto que el de los hombres (56.4). Aunque es importante resaltar que el resultado más bajo fue obtenido por una mujer y que la cantidad de mujeres en esta población era excesivamente baja.

c. Para algunos niños, las afirmaciones como "Soy feliz" o "Soy una buena persona" eran demasiado ambiguas y consideraban que era difícil responder a estar preguntas con un sí o un no. 


\section{Discusión}

A partir de esta pequeña población, podemos mostrar estos resultados preliminares, con la finalidad de continuar esta investigación en el futuro ${ }^{23}$.

- El autoconcepto de estos niños con altas capacidades tiende a ser igual al de los niños normales. Sin embargo, existen grandes picos en los resultados, es decir, que hay niños con autoconceptos excesivamente bajos y otros muy altos. Lo que lleva a la conclusión de que mientras la mayoría de los niños con altas capacidades tienen un autoconcepto acorde a los demás niños de su edad, para algunos, éste puede ser muy alto o muy bajo.

- Los niños de la población estudiada pertenecen a un programa de enriquecimiento, en el que tienen la oportunidad de convivir con otros niños de su edad; se supone que esto permite que se desarrolle un mejor autoconcepto.

- Dos de los niños tenían autoconcepto muy bajos y dos el autoconcepto muy alto. Esto se puede relacionar con su personalidad y con su propio desarrollo afectivo. Sin embargo, es importante resaltar que los dos que fueron muy altos pertenecían a niñas.

- El autoconcepto intelectual de estos niños con altas capacidades es normal al de los demás niños de su edad. Sin embargo es importante resaltar que en aquellas preguntas que hacían referencia a sus capacidades, eran muy 
positivos; pero cuando esta capacidad estaba en relación con los demás, era bajo. Por lo que se concluye que los niños con altas capacidades poseen un mejor autoconcepto intelectual, si se refiere a sí mismos, pero no es así cuando depende de los demás.

- En cuanto a la autoestima, en estos niños con altas capacidades tiende a ser alta, es decir que están a gusto consigo mismos y en relación a sus logros. Aunque en este estudio, las dos personas que tuvieron resultados muy bajos, hicieron que el resultado global bajara.

- Para muchos niños es difícil responder a preguntas que son altamente abstractas, sobre todo las relativas a la felicidad, la bondad o el bienestar, porque no lo consideran un absoluto sino como relativo.

- Es notable que el autoconcepto físico de estos niños es más bajo que lo normal, por lo que se concluye que éste es un factor que sí se ve afectado en esta población de niños con altas capacidades, sobre todo si se considera que la mayoría de los examinados eran niños. Esto puede deberse a la preponderancia de su desarrollo intelectual o al sedentarismo que se propicia actualmente.

- En cuanto a los aspectos que pueden mejorarse se encuentran: el tamaño de la población, ya que siendo la población extremadamente reducida, los resultados no pueden ser generalizados; el equilibrio entre niños o niñas, ya que se encontró que el autoconcepto de las mujeres es ligeramente superior y sería importante que en un estudio futuro se comprobara esta información. 
Como resultado general, puede afirmarse que el autoconcepto de los niños con altas capacidades que fueron estudiados, es semejante al de los demás niños de su edad. Sin embargo, existen casos que salen de la media en extremo y que demandan especial atención, porque los niños hallados por debajo de la media pueden presentar situaciones problemáticas.

Este estudio encontró un resultado diverso al de su hipótesis, esto se puede relacionar con el hecho de que se trata de niños que asisten a un programa de enriquecimiento por lo que reciben atención especializada. Los resultados dan pie a futuras investigaciones en las que se sugiere aumentar la población a estudiar. 


\section{REFERENCIAS BIBLIOGRÁFICAS}

ACEREDA, A., Niños superdotados., España, Editorial Pirámide., 2000., 275 págs.

BURNS, R. B., El autoconcepto: teoría, medición, desarro1 lo y comportamiento., España, Ediciones EGA., 1990., 355 págs.

CLARK, B., Growing up gifted., Estados Unidos de América, Prentice Hall., 1992., 607 págs.

GARDNER, H., Inteligencias múltiples: la teoría en la práctica., España, Paidós., 1995., 313 págs.

HARRIS, D.B. y PIERS, E.V., The Piers Harris chidren's concept scale., Estados Unidos de América., Western Psychological Services., 1969.

LYNCH, M. et al., Self-concept Adavances in theory and research., Estados Unidos de América., Ballinger Publishing Company., 1981., 367 págs.

LOVECKY, D., "Exploring social and emocional aspects of giftedness in children", en Roeper Review., Vol. 15, No.1., pp.34-46.

ONATE, M., El autoconcepto: formación medida e implicaciones en la personalidad., Madrid., Narcea., 1989., 191 págs.

JAMES, W., The principles of psychology., Estados Unidos de América., Dover Publications., 1980., 626 págs.

PAPALIA, D., Psicología del desarrollo de la infancia a la adolescencia., México., McGraw Hill., 1992., 672 págs.

PÉREZ, L. et al., Educar hijos inteligentes., España., Editorial CCS., 2000., 176 págs.

PÉREZ, L., 10 palabras clave en superdotados., Navarra., Editorial Divino Verbo., 1993., 332 págs. 
PRIETO, M., Identificación, evaluación y atención a la diversidad del superdotado., España., Ediciones Aljibe., 1997., 206 págs.

RAIMY, V., The self-concept as a factor in counseling and personality organization., Estados Unidos de América., Office of Educational Services., 1948., 156 págs.

RENZULLI, J.S., What makes giftdness? A reexamination of the definition of the gifted and talented., Estados Unidos de América, Ventura County Superintendent of Schools Office., 1979., 179 págs.

RIMM, S., Keys to parenting the gifted child., Estados Unidos de América., Barrron's., 1994., 185 págs.

SCHMITZ, C., Managing the social and emotional needs of the gifted., Estados Unidos de América., Free Spirit Publishing., 1985., 155 págs.

SHEA, T., Educación especial: un enfoque ecológico., México., Mc Graw Hill., 1997., 523 págs.

SILVIA Y ORTIZ, M., El niño sobredotado: ¿Cómo orientarlo?., México., Edamex., 1995., 267 págs.

WEBB, J., Guiding the gifted child., Estados Unidos de América., Gifted Psychology Press., 1994., 266 págs.

WEBB, J., "Nurturing Social Emotional Development of Gifted Children", en [www.ericfacility.net]., 2000.

WINNER, E., Gifted Children: myths and realities., Estados Unidos de América., Basic Books., 1996., 449 págs.

SOCIEDAD ESPAÑOLA PARA EL ESTUDIO DE LA SUPERDOTACIÓN., [www.ucm.es/info/sees]. 